\title{
Effect of pore structure on capillary condensation in a porous medium
}

\author{
M. R. Deinert ${ }^{1}$ and J-Y. Parlange ${ }^{2}$ \\ ${ }^{1}$ Department of Mechanical Engineering, University of Texas at Austin, Austin, Texas 78752, USA \\ ${ }^{2}$ Department of Biological and Environmental Engineering, Cornell University, Ithaca, New York 14853, USA
}

(Received 22 May 2008; revised manuscript received 9 November 2008; published 10 February 2009)

\begin{abstract}
The Kelvin equation relates the equilibrium vapor pressure of a fluid to the curvature of the fluid-vapor interface and predicts that vapor condensation will occur in pores or irregularities that are sufficiently small. Past analyses of capillary condensation in porous systems with fractal structure have related the phenomenon to the fractal dimension of the pore volume distribution. Recent work, however, suggests that porous systems can exhibit distinct fractal dimensions that are characteristic of both their pore volume and the surfaces of the pores themselves. We show that both fractal dimensions have an effect on the thermodynamics that governs capillary condensation and that previous analyses can be obtained as limiting cases of a more general formulation.
\end{abstract}

DOI: 10.1103/PhysRevE.79.021202

PACS number(s): 65.20.- w, 47.10.-g, 47.11.St, 47.56.+r

\section{INTRODUCTION}

The Kelvin equation is broadly used to understand gas adsorption to surfaces, the increased solubility of fine particles, phase change, and the topology of porous systems and surfaces (e.g., [1,2]). The equation relates the equilibrium vapor pressure of a fluid to the curvature of the fluid-vapor interface and predicts that vapor condensation will occur in pores or irregularities that are sufficiently small. The structure of the surface, or pore space, is particularly important in this context and considerable work has been done using the Kelvin equation to model adsorption and condensation processes in systems that have fractal structure (e.g., $[3,4]$ ).

Past analyses of capillary condensation on surfaces, and in porous media, have shown that the amount of fluid, $N$, that absorbs onto a surface (or condenses into a pore space) is given by

$$
N \propto C\left[\ln \left(P_{0} / P\right)\right]^{-\lambda} .
$$

Here $C$ is a constant, $P_{0}$ and $P$ are the saturation and equilibrium vapor pressures, and $\lambda=3-D$ where $D$ is either the surface fractal dimension of the substrate or the fractal dimension of the pore volume distribution of a medium $[2,3,5-7]$. For a porous system $D$ can also be interpreted as the surface fractal dimension of the pore space. In fact, the two interpretations are equivalent if the pore space is assumed to be comprised of smooth pores of radius $r$ whose cumulative number $N_{r}$ is given by $N_{r}(r>R) \sim r^{-D}$ [8]. Experimental work, however, has shown that some porous systems can exhibit distinct fractal dimensions that are characteristic of either their pore volume or the surfaces of the pores themselves (e.g., [9-12]). This suggests that, in addition to a fractal pore size distribution, some porous systems can have a pore network whose surfaces have a roughness that is itself fractal. Recent work has also shown that both pore surface and pore volume fractal dimensions would have an effect on capillary pressures in porous media [13], which suggests that they should have an impact on the thermodynamics that govern capillary condensation as well.

In the present paper we extend the thermodynamic formulation of equilibrium capillary pressure [14] to the phenom- ena of capillary condensation in a system with distinct pore volume and pore surface fractal dimensions. The analysis shows that $\lambda=\left(3-D_{v}\right) /\left(3-D_{s}\right)$, with $D_{s}$ being the pore surface fractal dimension and $D_{v}$ the pore volume fractal dimension. This result reduces to the standard formulation, $\lambda=3$ $-D_{v}$, when $D_{s}=2$, where $D_{v}$ is then the single fractal dimension that characterizes the topology of the pore space.

\section{CAPILLARY CONDENSATION IN MULTIFRACTAL POROUS MEDIA}

We adopt the convention of Yin [2] that capillary condensation in a porous medium results in the sequential filling of pores from small size to large. The distribution of wetting fluid in a porous system has two limiting regimes. When capillary forces dominate, the wetting fluid will concentrate in the most highly curved surface irregularities or pores. When fluid-substrate forces dominate, the fluid is largely found in a uniform film that covers the substrate [15]. However, in general both forces will have an effect on the distribution of condensed fluid within a porous system and they can be expressed in terms of the interfacial energy of the fluid in contact with the respective surface (i.e., substrate or nonwetting fluid). For a situation such as this, it was previously shown that the pressure change across an interface between a wetting and a nonwetting fluid can be expressed using an equation for the internal energy of a fluid element, which, under equilibrium conditions, yields [14]

$$
\Delta P=-\sum \sigma_{w, i}\left(\partial A_{w, i} l \partial V_{w}\right)_{U, S, T}
$$

Here $\Delta P$ is the pressure change across the capillary interface, $A_{w, i}\left(\mathrm{~m}^{2}\right)$ is the interfacial area of wetting fluid in contact with the $i$ th nonwetting phase, $\sigma_{w, i}\left(\mathrm{~J} / \mathrm{m}^{2}\right)$ is the respective interfacial surface tension, $V_{w}\left(\mathrm{~m}^{3}\right)$ is the volume of the wetting fluid, and the derivative is taken at constant internal energy, entropy, and temperature.

In order to evaluate the derivatives in Eq. (2) a relation is required that gives the fluid interfacial area as a function of the fluid volume within the pores. For a system where the pore surfaces exhibit fractal structure we use Mandelbrot's 
relation between a self-similar fractal area and the volume that it encloses $[10,13]$ and p. 112 of $[16]$ :

$$
A \propto V^{D_{s} / 3} .
$$

Here $A$ is the surface area of a given pore, $V$ is the volume enclosed by that area, and $D_{s}$ is the surface fractal dimension of the pore with $2 \leqslant D_{s} \leqslant 3$ [13]. We assume that fluid volume and pore volume are equal to within a negligible error and that the interfacial areas $A_{w, i}$ adhere to the proportionality in Eq. (3), which can be made direct using $A_{w, i}$ $=f_{w, i} V^{D s / 3}$, where $f_{w, i}$ is a shape factor that is assumed to remain constant across all scales. Using this relation, it was previously shown that $\left(\partial A_{w, i} / \partial V_{w}\right)=f_{w, i}\left(D_{s} / 3\right) V^{(1 / 3)\left(D_{s}-3\right)}$, where $V$ is the volume of the largest pore filled [13].

The fluid volume within the pore system can be written as $V_{w}=\int V d N$ [17]. Here $V$ is the volume of a single filled pore of a given size, $N$ is the number of those pores within a representative elementary volume of the medium, and the integral is over the applicable size range. It is experimentally observed that a wide variety of porous systems have pore spaces where the pore volumes follow a cumulative distribution function where the number of pores with radius greater than $L$ is given by

$$
N_{1}(I>L)=F L^{-D_{v}} .
$$

Here $L$ is taken to be the pore "radius" that corresponds to $V$, $F$ is a scaling constant, and $D_{v}$ is the pore volume fractal dimension. Equations (2)-(4) can be combined to give [13]

$$
\theta_{s}-\theta=C\left[1-\left(P_{s} / P\right)^{\lambda}\right] .
$$

Here $\theta$ is the fluid content of the medium, $\theta_{s}$ is the fluid content when the medium is saturated, $P$ is the capillary pressure, $P_{S}$ is the capillary pressure at fluid saturation, and $C$ is an experimentally determined constant that is a characteristic of the medium and the fluid just as is the $C$ that appears in Eq. (1). The exponent $\lambda=\left(3-D_{v}\right) /\left(3-D_{s}\right)$ [13].

At constant temperature, and under equilibrium conditions, one can write the change in free energy of a curved surface as

$$
\Delta G=v \Delta P
$$

where $G$ is the Gibbs potential, $v$ is the fluid's molar volume, and $\Delta P$ is the change in pressure across the interface, which here is the same as the capillary pressure $P$ in Eq. (5). The difference in molar free energy above and below a curved fluid surface is itself given by

$$
\Delta G=\mu-\mu_{0}=R T \ln \left(P / P_{0}\right),
$$

where $\mu$ and $\mu_{0}$ are the chemical potentials of the curved and planar fluids, respectively, and $P$ and $P_{0}$ are the vapor pres- sures of the pure fluid in its curved and planar states. Combing Eqs. (5)-(7) we get

$$
-v P_{s}\left[1-\left(\theta_{s}-\theta\right) / C\right]^{-1 / \lambda}=R T \ln \left(P / P_{0}\right),
$$

where the minus sign results from the fact that the curvature of a wetting fluid within a capillary tube causes a wellknown decrease in the fluid's vapor pressure.

Equation (8) can be rearranged to get

$$
P=P_{0} \exp \left\{-\left[1-\left(\theta_{s}-\theta\right) / C\right]^{-1 / \lambda} v P_{s} / R T\right\} .
$$

When $C=\theta_{s}$ Eq. (5) gives the Brooks and Corey relationship between medium saturation and capillary pressure [18], and Eq. (9) reduces to

$$
P=P_{0} \exp \left[-v P_{s} / R T\left(\theta / \theta_{s}\right)^{-1 / \lambda}\right],
$$

which is similar to the previous formulation of capillary condensation in a porous medium but differs in the more general form of the exponent $\lambda$.

\section{CONCLUSIONS}

Equations (9) and (10) show that capillary condensation in a porous system is dependent on the fractal character of the pore space and the pore surfaces, as well as the interaction of the fluid with the medium and the nonwetting fluid phase. By considering a porous system with distinct pore surface and pore volume fractal dimensions we have arrive at a more general form of the exponent $\lambda=\left(3-D_{v}\right) /\left(3-D_{s}\right)$. When $D_{s}=2$, which corresponds to smooth pore surfaces, the analysis reduces to the standard relationship for capillary condensation, $\lambda=\left(3-D_{v}\right)$ in a porous system, as expected. Here $D_{v}$ is then the single fractal dimension that characterizes the topology of the pore space and is usually just given the symbol $D$. However, the result $\lambda=\left(3-D_{v}\right) /\left(3-D_{s}\right)$ shows that for porous systems with distinct surface and pore volume fractal dimensions, both are important to the capillary condensation process. The exact relationship, if there is one, between $D_{v}$ and $D_{s}$ in natural systems is still a matter of conjecture. Equations (9) and (10) also give an explicit relationship between the condensed fluid content of the porous system and the equilibrium vapor pressure of the uncondensed phase. However, the formulation for moisture content ignores the effect that formation of a uniform multilayer of fluid by adsorption on the substrate might have. For situations where this effect is significant, it would need to be taken into consideration.

\section{ACKNOWLEDGMENTS}

Special thanks are given to Jack Howell and Phil Schmidt for their comments and suggestions. 
[1] L. R. Fisher, R. A. Gamble, and J. Middlehurst, Nature (London) 290, 575 (1981).

[2] Y. Yin, Langmuir 7, 216 (1991).

[3] B. Sahouli, S. Blacher, and F. Brouers, Langmuir 12, 2872 (1996).

[4] J. M. Kate and C. S. Gokhale, Eng. Geol. (Amsterdam) 84, 48 (2006).

[5] D. Avnir and J. Mieczyslaw, Langmuir 5, 1431 (1989).

[6] A. P. Radlinkski et al., Org. Geochem. 31, 1 (2000).

[7] A. P. Radlinkski et al., Int. J. Coal Geol. 59, 245 (2004).

[8] P. Pfeifer and D. Avnir, J. Chem. Phys. 79, 3558 (1983).

[9] P. Wong and Q. Cao, Phys. Rev. B 45, 7627 (1992).

[10] D. Gimenez et al., Geoderma 77, 19 (1997).
[11] F. Stallmach et al., Phys. Rev. Lett. 88, 105505 (2002).

[12] A. Dathe and M. Thullner, Geoderma 129, 279 (2005).

[13] M. Deinert, A. Dathe, J. Y. Parlange, and K. B. Cady, Phys. Rev. E 77, 021203 (2008).

[14] M. R. Deinert, J. Y. Parlange, and K. B. Cady, Phys. Rev. E 72, 041203 (2005).

[15] D. Broseta, L. Barre, and O. Vizika, Phys. Rev. Lett. 86, 5313 (2001).

[16] B. Mandelbrot, The Fractal Geometry of Nature (Freeman, New York, 1983).

[17] E. Perrier et al., Water Resour. Res. 32, 3025 (1996).

[18] R. H. Brooks and A. T. Corey, Colorado State University Hydrology Paper No. 3, 1964 (unpublished). 